\title{
Robust Design Based Optimisation of Waterjet Cutting
}

\author{
Tudor Deaconescu \\ Transilvania University of Brasov, Industrial Engineering and Management Department, Faculty of Technological Engineering and Industrial \\ Management, Romania
}

\begin{abstract}
The most important input quantities of waterjet cutting are the jet pressure, feed speed, stand-off distance, abrasive graining, mass flow, etc. Other quantities contributing to machining efficiency are the type of utilized abrasive or the tilt of the jet. Each of these quantities can be assigned different set points. The roughness of the machined surfaces and the thickness of the cut part are output quantities of the system, their values depending on the input parameters and the influence of various disturbing factors (noises). The paper discusses surface roughness obtained consequently to abrasive jet cutting. Optimisation of the machining system was achieved by intervening on five selected input quantities (factors), with two set points considered for each. Upon applying Taguchi methods, eventually the combination of factor set points was determined that ensures robust behaviour of the system.
\end{abstract}

\section{Introduction}

Waterjet cutting has proved its utility due to its versatility and easy deployment. The benefits that ensure waterjet abrasive machining its top position among special technologies are the diversity of machinable materials at low cost, reduced environmental impact, high flexibility, easy programming, rapid machining, and conservation of the material's properties due to the low processing temparatures. All these advantages call for in-depth research and swift solving of the problems posed by waterjet cutting: relatively reduced dimensional accuracy of the products (about $0.1 \mathrm{~mm}$ ) and the occurrence of certain defects (striations, mini-craters, high surface roughness).

The relatively low machining cost as well as the minimum impact on the environment render waterjet erosion a technology of the future. Emerged and refined over the last years, abrasive waterjet microprocessing has succeeded in improving processing precision to $0.02 \mathrm{~mm}$, while surface roughness can be optimised by means of adequately configuring the influential input factors of the process.

One of the methods of abrasive erosion of parts is waterjet machining or waterjet cutting, where erosion is initiated by the kinetic energy of a moving fluid. Waterjet as an erosive agent (without added abrasive) dates back as far as the 1900, when it was used in mining for cutting coals at pressure not exceeding $100 \mathrm{MPa}$.

The abrasive jet machining technology entails introducing a certain quantity of abrasive grains into the waterjet, thus improving its coherence, amplifying its mechanical effect and increasing cutting speed by about $30 \%$. In waterjet machining erosion is activated by the kinetic energy of a fluid in motion.
Waterjet machining has become possible in the 1970s with the launching of dedicated pumps [1], [2]. The first cutting installations were developed allowing increased performance of machining. Further milestones in the evolution of this technology are the abrasive mixing tubes made by ROCTEC, a ceramic composite of tungsten carbides (1980), the fluid motion control systems (1990), the tilting system of the cutting head (2000) and the CNC software implemented on waterjet cutting machines in the first decade of the $21^{\text {st }}$ century [3].

Due to its numerous advantages, in many industrial countries this process has been employed intensively. Thus, for example in Germany abrasive jets were employed for the cutting of aluminium (41\%), steel $(29 \%)$, glass $(22 \%)$, granite, marble and rock $(21 \%)$, nonferrous metals $(7 \%)$, wood $(7 \%)$, rubber $(5 \%)$, titanium $(5 \%)$, etc. Abrasive jet cutting devices currently hold about $35 \%$ of the total cutting machines worldwide, what proves once again the efficiency and advantages of this processing method.

Due to their benefits, waterjet machining procedures are continuously expanding. The Global Waterjet Machine Market report for 2015-2019 highlights the waterjet machining technology sales forecast for the American market [4]. Thus, starting from sales of USD 781.16 million in 2014, an increase up to USD 1221.12 million is envisaged until 2019. The main beneficiaries are the aerospace, the automotive and the rock and ore extractive industries.

Figure 1 shows the current market shares of the most important manufacturers of waterjet machines as established in the mentioned report [4].

The mentioned report also estimates a number of about 20,000 waterjet machines deployed worldwide in 2014 and an increasing trend of 5-6\% yearly. 


\section{Market share}

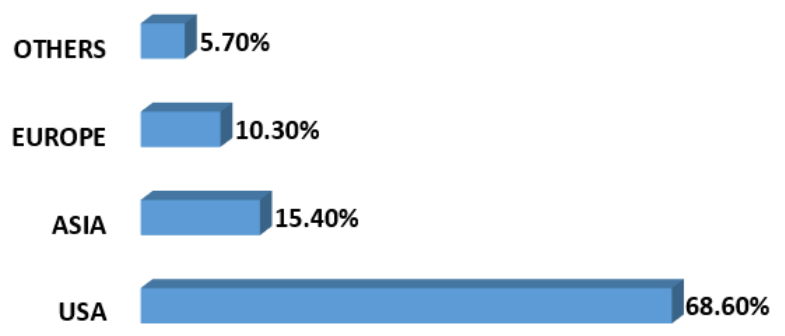

Figure 1. Current market shares of the top waterjet machine manufacturers.

The same report presents the increase of the market due to the benefits of the technology and forecasts sales exceeding a billion dollars starting 2018 (Figure 2).

Sales value ( million \$)

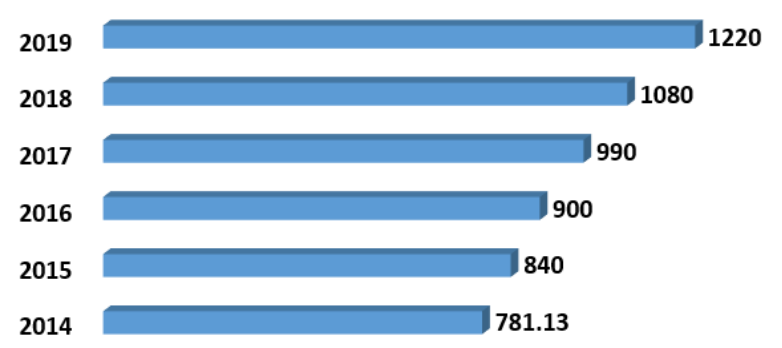

Figure 2. Annual sales values of waterjet machines

Pressure waterjet machining yields a number of significant benefits, including:

- special quality of the edges resulted upon cutting, eliminating the need for subsequent deburring;

- the cut contour can have complex shapes; zig-zag lines can be machined, as well as narrow bridges and interior contours;

- depending on the diameter of the jet, a very narrow cutting slot is generated in the part, thus achieving important material savings;

- tool sharpening and re-sharpening operations are eliminated;

- no preliminary contour drawing on the surface of the part is necessary;

- the machining process lends itself to automation;

- high productivity of cutting;

- minimum processing expenditure, given the reduced cost of water, abrasives, etc.

In relation to the latter advantage, Table 1 features the hourly costs of an abrasive jet cutting installation.

Table 1. Costs generated by abrasive jet erosion.

\begin{tabular}{|l|c|}
\hline & {$[\%]$} \\
\hline Labour & 35 \\
Machine (amortisation in 5 years $-10^{4}$ hours) & 25 \\
Abrasives & 15 \\
Nozzle & 10 \\
Pump maintenance & 10 \\
Energy & 5 \\
Total & 100 \\
\hline
\end{tabular}

A comparison between waterjet cutting and cutting with welding equipment, with plasma, laser, abrasive stones or a mechanical saw highlights the benefits of the former, as cutting occurs at normal, even low temperatures and no alteration of the material structure are caused by melting or internal stress. The relatively low price and minimum impact on the environment render waterjet cutting a technology of the future. Emerged and developed over the last years waterjet micromachining has improved precision to $0.02 \mathrm{~mm}$ and allows optimisation of surface roughness by configuring the process input factors by robust design [5].

An exact analysis of the mechanism of pressure abrasive fluid jet erosion is difficult, due to the multitude of individual processes which take place at the contact between the incident beam and the processed surface. The complexity of the process results from the effects of impact pressures, compression and shearing stresses, erosion and cavitation, all combined with the various properties of the processed materials.

Abrasive jet erosion is not "cutting" in the sense of carving, but a chip removal process, not unlike saw cutting. Analysis of material removed by an abrasive jet reveals chips of up to $5 \mu \mathrm{m}$, comparable to those generated in cutting with a diamond disk.

Machining by pressure abrasive fluid jets entails, in a first stage calibration of the fluid in the nozzle. In the second stage the fluid-abrasive mix is formed, which is sent towards the surface of the workpiece. It is extremely difficult to observe the forming process of the abrasive mix, because of the high speed of the grains $(200 \ldots 800$ $\mathrm{m} / \mathrm{s}$ ) and their small dimensions. Figure 3 illustrates the stages of abrasive jet forming, and presents the main quantities contributing to the erosive process.

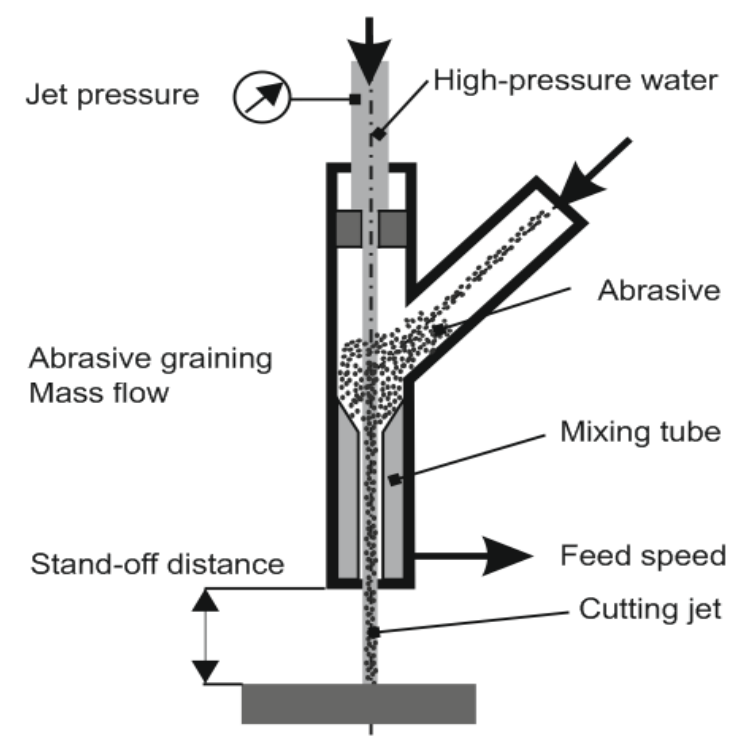

Figure 3. Principle of abrasive jet cutting.

The above figure shows that the abrasive grains are transported to the mixing tube by aspiration, via the water jet. Once in the mixing tube, the grains repeatedly collide with its walls and the water jet, being eventually included in the liquid jet. Because of these repeated collisions, the abrasive grains are continuously crushed, so that they 
have significantly smaller dimensions when leaving the mixing tube and hitting the workpiece.

Particularly significant is the form of the grains utilized in abrasive jet machining. Grain shape should be as compact and close to spherical as possible, as illustrated in Figure 4.

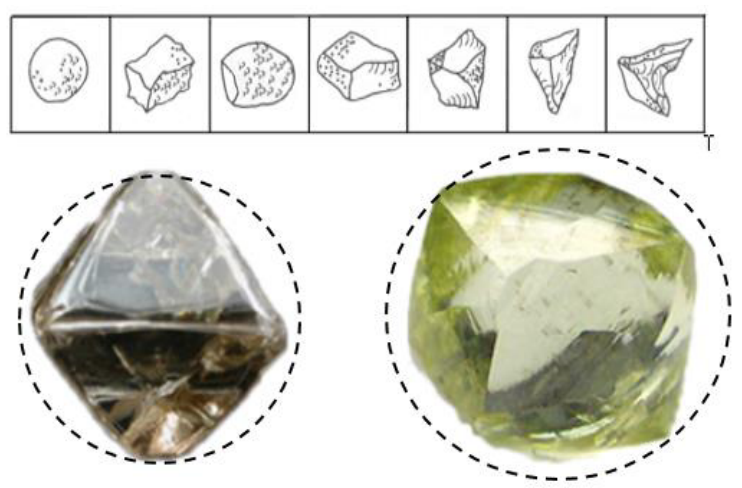

Figure 4. Recommended abrasive grain shapes.

The abrasive grains used in waterjet machining are selected by a form factor calculated by equation:

$$
F_{f}=\frac{d_{\min }}{d_{\max }}
$$

where $d_{\text {min }}$ is the largest diameter of a circle that can be inscribed in the abrasive grain, while $d_{\max }$ is the smallest diameter of a circle circumscribed to the grain. The usual values of the form factor range between 0.64 and 0.70 .

When leaving the mixing tube, the abrasive jet is characterised by four important properties: speed, coherence, density and symmetry. Speed is the most important characteristic of a jet, as it determines its cutting capacity. A higher speed improves capability of the jet to perform as a cutting tool.

Jet speed depends also on the stand-off distance; evidently jet speed decreases as the workpiece surface is located at a greater distance. Figure 5 presents jet speed variation versus stand-off distance, for a pressure of 200 $\mathrm{MPa}$ and an initial jet diameter of $0.2 \mathrm{~mm}$ [6].

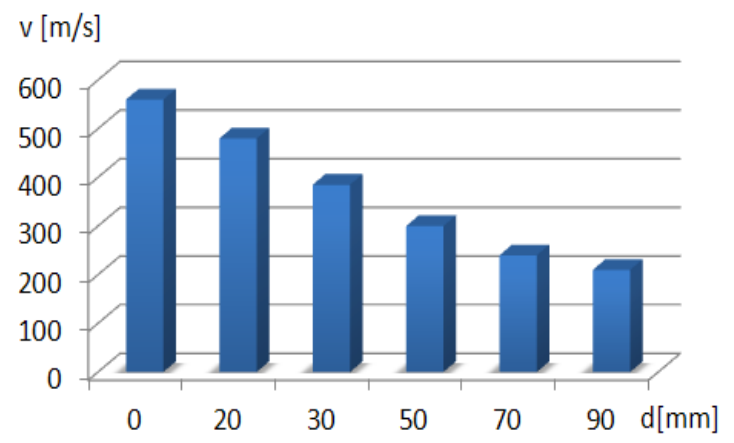

Figure 5. Jet speed versus stand-off distance.

As the jet penetrates the material, its kinetic energy decreases continuously, causing a curved trajectory of the cut (Figure 6). This disadvantage can be compensated by increasing jet speed $v$, or by decreasing the feed speed $w_{a}$. In general, a cut of high quality requires adequate correlation of jet speed, workpiece thickness and material.
Jet feed speed $w_{a}$ is particularly important for the quality of the cut. A higher feed speed reduces machining quality, and in some situations, when certain critical values are exceeded, the jet is deviated and cutting does not occur.

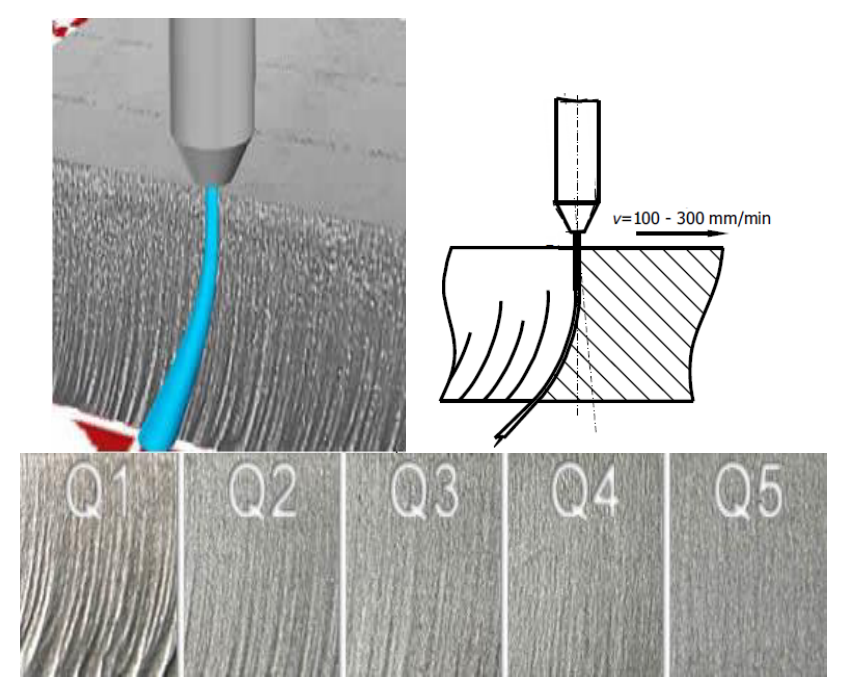

Figure 6. Curved trajectory of the cut.

In the above figure the quality of the cut is classified into five categories. While Q1 stands for a low quality cut yielding high surface roughness, Q5 indicates a cut achieved upon adequately setting the working parameters.

The mass flow of the abrasive grains is a further significant parameter for assessing the cutting capacity of the abrasive jet. Figure 7 presents the dependency of cut material thickness on the abrasive mass flow.

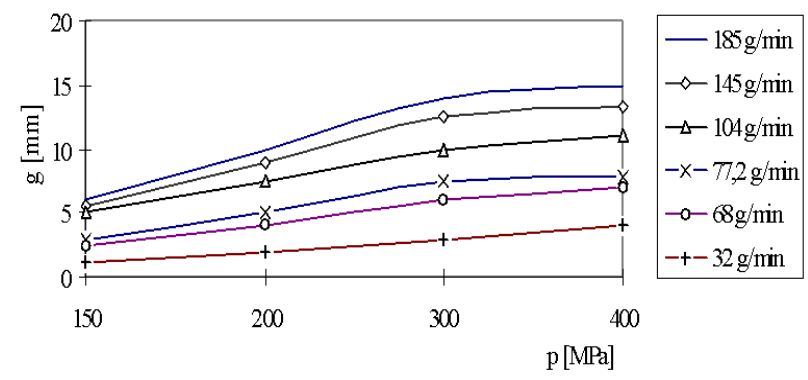

Figure 7. Influence of abrasive mass flow on cut thickness.

Erosion cutting by abrasive jets is achieved consequently to the cumulated effect of two phenomena:

- cutting and scraping carried out by each abrasive grain, during the first part of the machining process, as long as each grain still has sharp tips and cutting edges;

- $\quad$ plastic deformation of the material during the second part of the machining process, once the grains have lost their cutting capability. In this phase the remaining kinetic energy of the abrasive is still great enough to exceed to flow limit of the material, and thus in time to cause the removal of the tooling allowance.

Because of these two successive phenomena the quality of the cut surfaces is not uniform along the entire machined depth. A first area with numerous narrow striations can be observed, followed by an area with 
wider scratches (Figure 8). The depth of the first area, with a better roughness, depends directly on the speed of the abrasive jet.

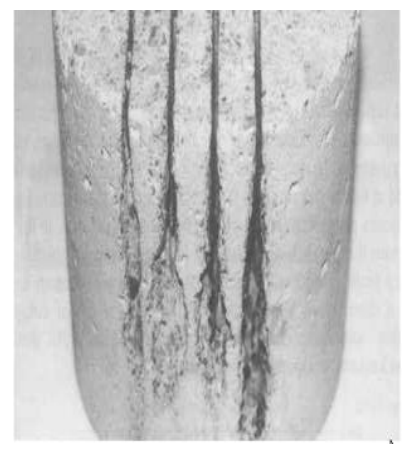

Figure 8. Width variation of the trace left by an abrasive grain.

It is known that as each abrasive grain penetrates deeper into the machined material, the quality of the cut decreases. Hence it is recommended to select the set points for the working parameters (pressure, graining, mass flow, feed speed) consequently to thorough experimental study. The thickness of the material that can be cut while ensuring acceptable quality needs to be determined by experiment-based multi-criteria optimisation.

Following analysis of the abrasive jet erosion process and knowing the main factors that contribute to obtaining surfaces of acceptable quality, further on a novel approach to ensuring machined surface quality is presented, based on the concept of robust design of these technological systems. This approach allows implementation of quality by conducting specially designed experiments of short duration, at low costs and with minimum material consumption.

The fundamental difference to classical R\&D of a system consists in the pragmatism of robust design that does not aim at identifying and eliminating the causes responsible for non-quality, considered "noises", but only render the system insensitive to their action.

\section{Experimental set-up}

Experimental studies for the robust optimisation of pressure abrasive jet machining were conducted on a Maxiem 1530 machine. Such a piece of equipment allows the cutting of workpieces of up to $3099 \mathrm{~mm} \times 1575 \mathrm{~mm}$, at a maximum available pressure of 3450 bar and a maximum feed speed of $9 \mathrm{~m} / \mathrm{min}$. The dimensional precision ensured by this machine is of $0.05 \mathrm{~mm}$.

The main parameters that contribute to attaining the goals of abrasive jet cutting are the pressure provided by the equipment, feed speed, stand-off distance, abrasive graining and mass flow. Other quantities impacting on machining efficiency are the type of the utilized abrasive and the tilting angle of the jet. All enumerated parameters represent input quantities of the system, each having a range of possible values.

Experimental research consisted of cutting workpieces made from an aluminium alloy with the following composition: $\mathrm{Al}$ : $92.02 \%$; $\mathrm{Cu}: 1.65 \%$; $\mathrm{Mg}$ : $2 \%$;
Cr: $0.23 \%$; Zn: $4 \%$; Mn: $0.1 \%$. The roughness $\left(R_{a}\right)$ of the surface was measured after testing.

The data sets obtained upon experimenting and measuring were processed by dedicated software, LappMaster, which yielded the optimum set-points of the tested controllable input factors.

\section{Experimental design}

Robust design of manufacturing systems is a major contribution brought by Japanese quality expert Genichi Taguchi to the set of instruments available in industry for quality analysis and improvement. Robustly designed processes ensure a constant quality, by reducing the scattering of the values measured for the quality characteristic. Significantly for this method, this desideratum is achieved without eliminating the causes responsible for variability, namely by rendering the process insensitive to the influence of such uncontrollable factors (noise factors).

Robust design is a method to reduce the interval of variability of the quality characteristics, by providing the methodology for systematically obtaining solutions that render manufacturing processes less sensitive to disturbing causes. This type of design can be utilized for optimising product development, as well as for conceiving new manufacturing processes.

According to Taguchi's strategy non-quality can be eliminated without eliminating the disturbing factors from the system, but by adequately combining the setpoints of the input factors.

The method used to optimize the pressure abrasive jet cutting process was the method of fractioned orthogonal factorial arrays of experiments devised by G. Taguchi. This method entails a planned approach, aimed at studying and establishing the links between the controllable input factors of a system and the obtained effects. The method identifies the combination of setpoints of the input parameters of the system that reduce the effects of parasite causes, without however addressing these directly.

From the numerous input quantities affecting the pressure abrasive jet cutting process five factors were selected considered to have a major contribution to the machining process. Table 2 presents these factors, each being assigned two set-points during experiments.

Table 2. Selected factors and associated set-points.

\begin{tabular}{|c|c|c|c|c|c|}
\hline \multirow{3}{*}{ 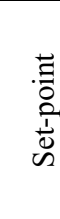 } & \multicolumn{5}{|c|}{ Factors } \\
\hline & $\begin{array}{c}\text { A } \\
\text { Feed } \\
\text { speed }\end{array}$ & $\begin{array}{c}\text { B } \\
\text { Jet } \\
\text { pressure }\end{array}$ & $\begin{array}{c}\mathrm{C} \\
\text { Stand- } \\
\text { off } \\
\text { distance }\end{array}$ & $\begin{array}{c}\mathrm{D} \\
\text { Abrasive } \\
\text { graining }\end{array}$ & $\begin{array}{c}\text { E } \\
\text { Mass } \\
\text { flow }\end{array}$ \\
\hline & {$[\mathrm{mm} / \mathrm{min}]$} & [bar] & {$[\mathrm{mm}]$} & {$[\mu \mathrm{m}]$} & {$[\mathrm{g} / \mathrm{s}]$} \\
\hline 1 & 50 & 1250 & 1 & 60 & 0.5 \\
\hline 2 & 100 & 1750 & 1.5 & 90 & 2 \\
\hline
\end{tabular}

If a full array of experiments were used, the total number of tests would be $2^{5}=32$. Such an array entails the experimental study of all possible combinations of the set-points of the considered input factors, influencing 
system operation. Not all tests have, however, the same practical significance, some of the runs providing more information than others. Starting from this observation, G. Taguchi has devised a set of fractioned orthogonal factorial arrays of experiments. These arrays allow considerable reduction of the number of test runs to be conducted, without diminishing relevance and accuracy of the yielded results.

The experiments were conducted according to an array of experiments requiring only 8 runs, each with a different combination input factor set-points.

Table 3 presents this array of experiments, indicating in brackets, next to the set-point for each factor, the respective concrete values.

Table 3. The utilized array of experiments.

\begin{tabular}{|c|l|l|l|l|l|}
\hline \multirow{2}{*}{ Run } & \multicolumn{5}{|c|}{ Factors } \\
\cline { 2 - 6 } & A & B & C & D & E \\
\hline 1 & $1(50)$ & $1(1250)$ & $1(1)$ & $1(60)$ & $1(0.5)$ \\
\hline 2 & $1(50)$ & $1(1250)$ & $1(1)$ & $2(90)$ & $2(2)$ \\
\hline 3 & $1(50)$ & $2(1750)$ & $2(1.5)$ & $1(60)$ & $1(0.5)$ \\
\hline 4 & $1(50)$ & $2(1750)$ & $2(1.5)$ & $2(90)$ & $2(2)$ \\
\hline 5 & $2(100)$ & $1(1250)$ & $2(1.5)$ & $1(60)$ & $2(2)$ \\
\hline 6 & $2(100)$ & $1(1250)$ & $2(1.5)$ & $2(90)$ & $1(0.5)$ \\
\hline 7 & $2(100)$ & $2(1750)$ & $1(1)$ & $1(60)$ & $2(2)$ \\
\hline 8 & $2(100)$ & $2(1750)$ & $1(1)$ & $2(90)$ & $1(0.5)$ \\
\hline
\end{tabular}

Each run was repeated five times, and roughness $R_{a}$ was measured in different areas of the machined surface. Further arithmetic mean, standard deviation and signalto-noise ratio were computed for the measured results.

The signal-to-noise ratio $(\mathrm{S} / \mathrm{N})$ is the performance indicator that simultaneously considers the target value of the analysed quality criterion and the variability around the target. The desired value is defined as signal (expressed by $\bar{y}=$ the arithmetic mean of the measured values), while its undesired variability, to be diminished, is defined as noise, (expressed by the standard deviation $s)$.

In certain practical applications merely determining the results of measurements may not be sufficient. In the case of pressure abrasive jet cutting, one of the aims is to machine surfaces such as to achieve a roughness as small of possible. This means imposing a sense of this quantity's evolution, in other words considering an optimisation criterion of "smaller is better" type. The computational relationship of the $\mathrm{S} / \mathrm{N}$ ratio for a quality characteristic whose value, in order to be optimum, should tend towards a minimum, as is the case of surface roughness is:

$$
S / N_{m}=-10 \cdot \log \left(s^{2}+\bar{y}^{2}\right)
$$

Obviously, the higher the algebraic value of the signal-to-noise ratio is (maximised signal - to minimized noise), the better the performance of the machining system will be. Consequently, based on the results obtained by running the LappMaster programme the optimum set-points for each analysed input factor could be established. Table 4 presents these results.
Table 4. Optimum combination of factor set-points.

\begin{tabular}{|c|l|c|c|}
\hline Factor & Factor description & $\begin{array}{c}\text { Optimum } \\
\text { set-point }\end{array}$ & $\begin{array}{c}\text { Value of } \\
\text { optimum set- } \\
\text { point }\end{array}$ \\
\hline A & Feed speed & 1 & $50 \mathrm{~mm} / \mathrm{min}$ \\
\hline B & Jet pressure & 1 & $1250 \mathrm{bar}$ \\
\hline C & Stand-off distance & 2 & $1.5 \mathrm{~mm}$ \\
\hline D & Abrasive graining & 1 & $60 \mu \mathrm{m}$ \\
\hline E & Mass flow & 1 & $0.5 \mathrm{~g} / \mathrm{s}$ \\
\hline
\end{tabular}

By setting the values of the five factors to the determined points, the expected resulting signal-to-noise ratio is of $-6.4276 \mathrm{~dB}$, which value would generate a robust abrasive jet cutting process. This value of resulting $\mathrm{S} / \mathrm{N}$ ratio was computed according to equation (3):

$$
\frac{S}{N}_{\text {res }}=\frac{\bar{s}}{N}+\sum \text { the effects with the highest alg.value }
$$

\section{Conclusion}

The paper presents a study of high pressure abrasive jet cutting, optimised by the method of robust parameter design. This approach is aimed at rendering the analysed system insensitive to the action of noise factors, by an adequate combination of the input factor set-points.

In the first stage of applying the method of fractioned orthogonal factorial arrays of experiments devised by G. Taguchi, five controllable input factors were selected, and two set-points were considered for each. Within the ensuing phase, a set of experiments were run, with a setpoint assigned to each factor as provided by Taguchi's fractioned orthogonal factorial array; eventually, upon mathematical processing of the measured results a combination of optimum set-points of the analysed factors resulted. By assigning these optimum set-points to the input quantities the machining system becomes robust, and consequently the roughness of the machined surfaces will not deviate from the desired and predicted value.

\section{References}

1. P.W. Bridgman, The Physics of High Pressure, (Dover Publications Inc., New York., 1970)

2. A.W. Momber, R. Kovacevic, Principles of abrasive waterjet machining, (Springer Verlag, London, 1998)

3. B. Barabaş, Current State of Research on Waterjet Machining of Surfaces, (Transilvania University of Braşov, 2015)

4. Report Buyer - Global Waterjet Machine Market 2015-2019, accessed November 2015, https://www.reportbuyer.com/product/869256/global -waterjet-machine-market-2015-2019.html

5. T. Deaconescu, Contributions to the abrasive waterjet machining of parts, Nonconventional Technologies Rev., 4 (2015)

6. C. Birtu, Contributions to Materials Machining by Hydro-Abrasive Jets, (Doctoral Thesis, Bucureşti, 2000) 